SU-TS-9502

\title{
Insulator-to-metal transition in Kondo insulators under strong magnetic field *
}

\author{
Tetsuro Sasoli and Masatoshi Itoh* \\ Department of Physics, Faculty of Sciences, Saitama University, Urawa, 338 Japan
}

(September 5, 1995)

\begin{abstract}
Magnetization curve and changes of the single-particle excitation spectra by magnetic field are calculated for the periodic Anderson model at half-filling in infinite spatial dimension by using the exact diagonalization method. It is found that the field-induced insulator-to-metal transition occurs at a critical field $H_{c}$, which is of the order of the single ion Kondo temperature. The transition is of first order, but could be of second order in the infinite system size limit. These results are compared with the experiments on the Kondo insulator $\mathrm{YbB}_{12}$.
\end{abstract}

PACS numbers: 05.30.Fk, 71.28.+d, 75.20.Hr

Typeset using REVTEX

*To appear in Phys. Rev. B 
Study of the strongly correlated electron systems is one of the main issues in condensed matter physics. The heavy fermion materials [1] are the typical examples of such systems, in which the conduction electrons mix with the almost localized $4 \mathrm{f}$ or $5 \mathrm{f}$ electrons, and form the strongly renormalized quasi-particles which have effective mass of 100 to 1000 times larger than the bare value. This strong renormalization is mainly due to the local Kondo-type processes, which should be suppressed if an energy gap opens at the Fermi level.

Recently, the materials called Kondo insulators are attracting much interest. SmS [2], $\mathrm{SmB}_{6}$ [3], $\mathrm{YbB}_{12}$ [4], TmSe [5], $\mathrm{Ce}_{3} \mathrm{Bi}_{4} \mathrm{Pt}_{3}$ [6], CeNiSn [7], etc. belong to this category. These materials are considered to be the intermediate-valence systems [1], but show the activation type increase of the resistivity at low temperatures, leading to insulating ground states, instead of the metallic behaviors in the ordinary heavy fermion or valence-fluctuating materials. In these Kondo insulators, a gap opens at Fermi level because of the mixing of the conduction electrons with the periodic assembly of the localized electrons. The single-site Kondo processes, however, are still possible if the gap is comparable to or smaller than the Kondo temperature [8]. In addition, the Kondo processes reduce the mixing, so that the gap is renormalized to the value of the order of the Kondo temperature. Thus the Kondo effect and the renormalization of the gap take place in a self-consistent way. It is observed that the specific heat coefficient $\gamma$ (extrapolated from the temperatures higher than the gap) is enhanced to some extent in the Kondo insulators [7], which indicates an importance of the Kondo-type renormalization in these systems.

To further investigate the characters of the gap, an interesting experiment was done on $\mathrm{YbB}_{12}$ [9], in which the application of the strong magnetic field of up to 55T destroys the gap and the system goes a transition from insulator to metal. The magnetization starts to steeply increase at the critical field. These experiments stimulate us to understand how the above-mentioned states with strong renormalization will be modified or destroyed by the magnetic field from the theoretical point of view. For these purposes, we utilize the recently developed ideas and techniques to treat the strongly correlated electron systems in the infinite spatial dimensions [10]. We have calculated the single-particle excitation 
spectra to determine the energy gap, which vanishes at certain critical field $H_{c}$, indicating the insulator-to-metal transition, and the magnetization curves, which exhibits a steep rise at $H_{c}$. These results are consistent with the experiments on $\mathrm{YbB}_{12}$. Furtheremore, we have showed that the Van Vleck susceptibility is finite even in insulators, and is enhanced by the electron correlation.

We start with the periodic Anderson model (PAM),

$$
\begin{aligned}
H & =\sum_{i j \sigma} t_{i j} c_{i \sigma}^{+} c_{j \sigma}+\sum_{i \sigma} E_{f \sigma} f_{i \sigma}^{+} f_{i \sigma} \\
& +\frac{V}{\sqrt{N}} \sum_{i \sigma}\left(f_{i \sigma}^{+} c_{i \sigma}+\text { h.c. }\right)+U \sum_{i} n_{f i \uparrow} n_{f i \downarrow}
\end{aligned}
$$

where $E_{f \sigma}=E_{f}-\sigma h, h=\mu_{B} H$ and $H$ denotes the magnetic field. The other notations are standard. We neglect the orbital degeneracy and assume the symmetric case $E_{f}=-U / 2$ together with half-filling condition to express the simplest model to the Kondo insulators. Note that we apply magnetic field only to f-electrons. The reason for this is that the $g$ factor for f-electrons, $g_{f}$, differ from that for conduction electrons, $g_{c}$, so that the total magnetization is not a conserved quantity. The total susceptibility is given by $\chi=\left[\left(g_{f}-\right.\right.$ $\left.\left.g_{c}\right) / g_{f}\right] \chi_{f}$, where $\chi_{f}$ is the susceptibility in which the magnetic field is applied only to felectrons [1]. If we take $g_{f}=g_{c}$ and apply magnetic field to both electrons, $\chi$ vanishes for the half-filling at $T \rightarrow 0$, which apparently contradicts with experiments. We therefore calculate only $\chi_{f}$ and denote it simply as $\chi$ in the following. In more realistic models, one has to take account of the real values of $g$ factors for both electrons, and also the values of the magnetic moments in the crystal field split states [12.

The first term in eq.(1) denotes the kinetic energy of conduction electrons and is scaled in such a way that in $d \rightarrow \infty$ limit ( $d$ denotes the spatial dimensions) the density of states (DOS) has the form $\rho(\epsilon)=(2 / \pi W) \sqrt{1-(\epsilon / W)^{2}}$, where $W=2 \sqrt{d} t$, if the Bethe lattice with the hopping $t$ parameter is assumed. We, however, regard it as a model DOS for the real system. Furthermore, this shape is more suitable for the discussion of the insulatormetal transition, since it yields the sharp transition in contrast to the Gaussian DOS. We also assume in the following that any magnetic order is suppressed because of an insufficient 
nesting or strong frustrations in real systems, so that we study only the paramagnetic phase. $W=\hbar=k_{B}=1$ is assumed throughout the present paper.

We replace $E_{f}$ by $\tilde{E}_{f}=E_{f}+U / 2=0$ in the Hamiltonian, assuming the electron-hole symmetry, and add the term $(U / 2) \sum_{i \sigma} n_{\text {fio }}$ to compensate the above modification. But the latter term is constant because of the electron-hole symmetry even under finite field since $n_{f i \sigma}=\left(n_{f}+\sigma m\right) / 2$ and $n_{f}=1$, where $m=n_{f i \uparrow}-n_{f i \downarrow}$ defines the magnetization. The thermal Green's function for f-electrons is expressed as

$$
G_{f \sigma}\left(i \epsilon_{n}\right)=\int d \epsilon_{k} \frac{\rho\left(\epsilon_{k}\right)}{G_{f \sigma}^{0}\left(k, i \epsilon_{n}\right)^{-1}-\Sigma_{\sigma}\left(i \epsilon_{n}\right)}
$$

where $G_{f \sigma}^{0}\left(k, i \epsilon_{n}\right)=\left(i \epsilon_{n}-\tilde{E}_{f}+\sigma h-V^{2} /\left(i \epsilon_{n}-\epsilon_{k}\right)\right)^{-1}$ denotes the unperturbed f-Green's function. In $d \rightarrow \infty$, the lattice problem is reduced to solving a generalized Anderson impurity embedded in an effective medium self-consistently [13,15]. The unperturbed Green's function for this impurity is given by $\tilde{G}_{f \sigma}\left(i \epsilon_{n}\right)^{-1}=G_{f \sigma}\left(i \epsilon_{n}\right)^{-1}+\Sigma_{\sigma}\left(i \epsilon_{n}\right)$. We can fit $\tilde{G}_{f \sigma}\left(i \epsilon_{n}\right)^{-1}$ by that of the impurity Anderson model with finite number of conduction electron levels $\epsilon_{\ell}$ and energy-dependent mixing $V_{\ell}\left(\ell=1 \sim N_{s}\right): \tilde{G}_{f \sigma}^{f i t}\left(i \epsilon_{n}\right)^{-1}=\left(i \epsilon_{n}-E_{\sigma}+\sigma h-\sum_{\ell \sigma} v_{\ell \sigma}^{2} /\left(i \epsilon_{n}-\epsilon_{\ell \sigma}\right)\right)$ [16]. Accuracy of this fitting is generally very well in spite of the small number of levels. It is because not only the mixing $V_{\ell}$ 's but also the energy levels $\epsilon_{\ell}$ 's are treated as the fitting parameters. As a result, bulk quantities can be obtained with good accuracy in the present method, although discreteness of the levels reflects in dynamical quantities. $E_{\sigma}$ above denotes the effective $\mathrm{f}$ level, but the fitting always yields $E_{\sigma}=0$. Although their method is applicable also to the finite temperatures, we investigate only the zero temperature properties. The ground state of this Anderson model was determined by the modified Lanczos method [17] and the thermal Green's function $G_{f \sigma}\left(i \epsilon_{n}\right)$ was calculated in the form of the continued fraction by the Lanczos method [18]. It is known that $N_{s}=7$ is sufficient to obtain a good fit to the thermal Green's function by that with finite levels for the Hubbard model [16] and the same holds true in the present case. A new estimate of the self-energy is obtained by $\Sigma_{\sigma}\left(i \epsilon_{n}\right)=\tilde{G}_{f \sigma}\left(i \epsilon_{n}\right)^{-1}-G_{f \sigma}\left(i \epsilon_{n}\right)^{-1}$, which completes the self-consistent loop.

We first present the single-particle excitation spectrum for $U=2$ and $h=0$ in Fig.1(a). 
$V=0.5$ is used in the following calculations. It should be noted that the bare f-electron peaks at $\pm U / 2$ are shifted to deeper positions, in accord with the quantum Monte Carlo calculation [20], the numerical renormalization group calculation [21] and the self-consistent second order perturbation theory [11]. In addition to them, a Kondo resonance peak is formed around $\epsilon=0$, which is split by the mixing with conduction electrons. The size of the gap, $E_{g}$ are plotted in Fig.2 as a function of $U . E_{g}$ is about twice the Kondo temperature $T_{K}$ for the single impurity for large $U$. Here we define $T_{K}$ by $T_{K}=1 / 4 \chi$, where $\chi$ is the magnetic susceptibility obtained by Bethe Ansatz for the impurity Anderson model [14].

It should be noted that the self-energy $\Sigma_{\sigma}\left(i \epsilon_{n}\right)$ is proportional to $-i \epsilon_{n}$ even in the insulating phase. This is in contrast to the case of the Hubbard model, in which $\Sigma_{\sigma}\left(i \epsilon_{n}\right) \propto-i \epsilon_{n}$ in the metallic phase but $\propto-i / \epsilon_{n}$ in the insulating phase [15]. A reason to this difference would be that the gap is formed by the mixing in PAM and it exists even for $U=0$ whereas it is purely due to the correlation in the Hubbard model.

Therefore, the above spectra are well represented by the renormalized mean-field (MF) theory (eq.(5) below with $h=0$ ), in which $V^{2}$ in the mixed band dispersion

$$
E^{( \pm)}\left(\epsilon_{k}\right)=\frac{1}{2}\left[\epsilon_{k}+\tilde{E}_{f} \pm \sqrt{\left(\epsilon_{k}-\tilde{E}_{f}\right)^{2}+4 V^{2}}\right],
$$

is replaced with $z V^{2}$, where $z$ is the renormalization factor, $z^{-1}=1-\partial \Sigma_{\sigma}\left(i \epsilon_{n}\right) /\left.\partial i \epsilon_{n}\right|_{\epsilon_{n} \rightarrow 0}$. For $U=2$ we found $z=0.57$. The energy gap is given by $E_{g}=E^{(+)}\left(\epsilon_{k}=-W\right)-E^{(-)}\left(\epsilon_{k}=\right.$ $+W)=-W+\sqrt{W^{2}+4 z V^{2}}$ for the symmetric case. It reads $E_{g} \approx 2 z V^{2} / W$ when $V \ll W$.

Next, we apply magnetic field. Fig. 2 displays the magnetization curve of the f-moment $m=n_{f \uparrow}-n_{f \downarrow}$ for $\mathrm{U}=0,1,2$ and 3 . The dotted curve is the result for the infinite system with $U=0$ obtained from

$$
m=\int d \epsilon f(\epsilon)\left[\rho_{f \uparrow}(\epsilon)-\rho_{f \downarrow}(\epsilon)\right],
$$

where $f(\epsilon)$ is the Fermi function and $\rho_{f \sigma}(\epsilon)$ denotes the f-component density of states of $\sigma$ spin electrons under magnetic field,

$$
\rho_{f \sigma}(\epsilon)=\frac{1 \mp b_{\sigma}}{1 \pm b_{\sigma}} \rho\left(E_{\sigma}(\epsilon)\right)
$$


(upper (lower) sign for $\left.\epsilon>\tilde{E}_{f \sigma}\left(\epsilon<\tilde{E}_{f \sigma}\right)\right)$,

$$
b_{\sigma}=\left(E_{\sigma}(\epsilon)-\tilde{E}_{f \sigma}\right) / \sqrt{\left(E_{\sigma}(\epsilon)-\tilde{E}_{f \sigma}\right)^{2}+4 V^{2}}
$$

$E_{\sigma}(\epsilon)=\epsilon-V^{2} /\left(\epsilon-\tilde{E}_{f \sigma}\right)$ and $\tilde{E}_{f \sigma}=\tilde{E}_{f}-\sigma h$. This formula gives the $\mathrm{f}$ component susceptibility $\chi_{f}=0.76676$, which agrees with the initial slope of the curve for $U=0$ in Fig. 3.

For $U=0$, the spectrum consists of only a few sharp peaks due to the smallness of the system, so that the magnetization curve shows redundant steps, although its gross feature agrees well with eq.(四). For finite $U$, however, the spectra acquire finer structures and $m(h)$ becomes smoother, although a redundant two-step jump is still seen at $U=1$. Beside these artifacts, the magnetization curves indicate sharp increases at the critical field $h_{c}$, which is plotted in Fig. 2 as a function of $U$. The change of the spectra across $h_{c}$ are displayed in Fig. 1(b) $\left(h_{\sim}^{<} h_{c}\right)$ and (c) $\left(h_{\sim}^{>} h_{c}\right)$, indicating that the up- and the down- spin bands shift with each other and the gap closes. Thus the system undergoes a transition from insulator to metal at $h_{c}$.

We also show in Fig. 4 how the gap $E_{g}(h)$ closes by magnetic field. It is seen that the gap closes as $E_{g}(h)=E_{g}(0)-2 h$, but for $U>0$ (and maybe also for $U=0$ around $h=0.26$ ), $E_{g}$ jumps to zero before $E_{g}(0)-2 h$ vanishes, indicating a first order transition. By a naive argument we expect that the Kondo renormalization would be suppressed by the magnetic field, so that $z$ and the effective mixing increase, hence the gap increases, whereas the upand down-spin bands are relatively shifted by the field, so that the gap reduces. Hence the critical field $h_{c}$ is determined as a result of the competition of these two effects. The present calculation shows that the gap decreases in proportion to $-2 h$, which indicates that $z$ does not change but the gap is closed because of the rigid shift of the each spin band. In fact, we found that the self-energy scarcely changes its shape for $h<h_{c}$.

Whether the transition is of 1 st order is a delicate issue. Investigating the details of the spectra shown in Figs.1(b) and (c), we found that a small peak discontinuously moves across the chemical potential, $\epsilon=0$, when $h$ goes through $h_{c}$. Since we have electron-hole 
symmetry and even number of electrons in total, no state can appear precisely at $\epsilon=0$. Therefore, in the finite system calculation, a continuous change of $h$ across $h_{c}$ may cause a discontinuous shift of peaks across $\epsilon=0$, yielding a discontinuous closing of a gap. Thus, we expect that the transition could become second order in the infinite size limit, but we leave it open for further study.

Sugiyama, et al. [9] analysed their experiment on $\mathrm{YbB}_{12}$ and concluded that the gap decreases slightly faster than linearly with the field (see the insert in Fig.4). Since there are two activation energies in their resistivity data, they introduced a fine structure in the model density of states characterized by two gap values $2 \Delta_{1}=60 \mathrm{~K}$ and $2 \Delta_{2}=180 \mathrm{~K}$. The average of the two gives $E_{g} \sim 120 \mathrm{~K}$ and the critical field $H_{c} \sim 450 \mathrm{KOe}$ corresponds to 57 $\mathrm{K}$, using $g J=1.9$. Thus they satisfy $E_{g} \sim 2 H_{c}$. Our calculations are in good agreement to these features at least qualitatively.

Finally, we show in Fig.5 the f-component magnetic susceptibility obtained from the initial slopes of Fig.3, together with that multiplied by $E_{g}$ and $z$. Note that the susceptibility has finite values despite a finite gap opens, since the total magnetization is not conserved in the present model. It is sometimes called the Van Vleck susceptibility. Kontani and Yamada [22] gave a discussion that it should be enhanced approximately by $z^{-1}$. Our result shows that it is indeed enhanced by the interaction, although the enhancement becomes weaker than $z^{-1}$ or $E_{g}^{-1}$ for strong coupling regime, which may be due to the interspin exchange interaction. The present result is qualitatively in agreement with the self-consistent perturbational result [11].

In summary, we have calculated the magnetization curve and the single-particle excitation spectra of the periodic Anderson model at half-filling in infinite dimensions in the paramagnetic phase, which is a prototype of the Kondo insulators, and found that the insulator-to-metal transition takes place by applying magnetic field. The gap decreases as $E_{g}(h)=E_{g}(0)-2 h$, and vanishes at the critical field $h_{c}$, which is found to be of the order of $T_{K} / 2$. It indicates that the gap closes for $h<h_{c}$ in such a way that each spin band is shifted rigidly by $\pm h$. The transition is of first order, but could be of second order in the 
infinite system. The above calculation are mostly in accordance with the observations in $\mathrm{YbB}_{12}$, although more realistic model must be used for detailed comparison. Further study of the magnetic field effects to the states away from half-filling will also shed light on the metamagnetism in metallic heavy fermion systems [23].

This work is supported by Grant-in-Aid for Scientific Research on Priority Areas, No. 07233209, "Physics of Strongly Correlated Metallic Systems", from the Ministry of Education, Science and Culture. The computation was done using FACOM VPP500 in the Supercomputer Center, Institute for Solid State Physics, University of Tokyo. One of the authors (T. S.) thank Professor O. Sakai, Dr. K. Sugiyama and Mr. T. Mutou for useful conversations. 


\section{REFERENCES}

$\dagger \quad$ E-mail: saso@th.phy.saitama-u.ac.jp.

* $\quad$ Present address: CASIO Computer Co., Ltd., Hamura, Tokyo 190-11.

[1] For a review, see e.g. P. Fulde, J. Keller and G. Zwicknagl, Solid State Physics, vol.41, D. Turnbull and H. Ehrenreich (Eds.) (Academic Press, 1988).

[2] A. Jayaraman, V. Narayanamurthi, E. Bucher and R. G. Maines, Phys. Rev. Lett. 25 (1970) 1430.

[3] A. Menth, E. Buehler and T. H. Geballe, Phys. Rev. Lett. 22 (1969) 295.

[4] M. Kasaya, J. Mag. Magn. Mater. 47 \& 48 (1985) 429.

[5] P. Haen, F. Lapierre, J. M. Mignot, R. Tournier and F. Holtzberg, Phys. Rev. Lett. 43 (1979) 304.

[6] M. F. Hundley, P. C. Canfield, J. D. Thompson, Z. Fisk and J. M. Laurence, Phys. Rev. B42 (1990) 4862.

[7] T. Takabatake, F. Teshima, H. Fujii, S. Nishigori, T. Suzuki, Y. Yamaguchi, J. Sakurai and D. Jaccard, Phys. Rev. B41 (1990) 9607.

[8] J. Ogura and T. Saso, J. Phys. Soc. Jpn. 62 (1993) 4346.

[9] K.Sugiyama, F. Iga, M. Kasaya, T. Kasuya and M. Date, J. Phys. Soc. Jpn. 57 (1988) 3946.

[10] W. Metzner and D. Vollhardt, Phys. Rev. Lett. 62 (1989) 324.

[11] D. S. Hirashima and T. Mutou, Physica 199 \& 200 (1994) 206; J. Phys. Soc. Jpn. 63 (1994) 4475.

[12] M. Nakano, Phys. Rev. B44 (1991) 10300.

[13] M. Jarrell, Phys. Rev. Lett. 69 (1992) 168. 
[14] B. Horvatić and V. Zlatić, J. Physique 46 (1985) 1459.

[15] M. J. Rozenberg, X. Y. Zhang and G. Kotliar, Phys. Rev. Lett. 69 (1992) 1236.

[16] M. Caffarel and W. Krauth, Phys. Rev. Lett. 72 (1994) 1545.

[17] E. Dagotto and A. Moreo, Phys. Rev. B31 (1985) 865.

[18] E. R. Gagliano and C. A. Balseiro, Phys. Rev. Lett. 59 (1987) 2999.

[19] A. Georges and W. Krauth, Phys. Rev. B48 (1993) 7167.

[20] M. Jarrell, H. Akhlaghpour and Th. Pruschke, Phys. Rev. Lett. 70 (1993) 1670.

[21] O. Sakai, Butsuri 50 (1995) 615 (in Japanese).

[22] H. Kontani and K. Yamada, preprint (1995).

[23] J.-M. Mignot, J. Flouquet, P. Haen, F. Lapierre, L. Puech and J. Voiron, J. Mag. Magn. Mater. 76 \& 77 (1988) 97. 


\section{FIGURES}

FIG. 1. The single-particle spectrum of PAM for $U=2, V=0.5$ and (a) $h=0$, (b) $h=0.09$ and (c) $h=0.1$. The dotted line in (a) indicates the spectrum of the mean-field theory with reduced mixing $z V^{2}$ with $z=0.57$.

FIG. 2. The energy gap $E_{g}$, the twice of the critical magnetic field $2 h_{c}$ and the impurity Kondo temperature $T_{K}$ by the Bethe Ansatz are plotted against $U$.

FIG. 3. The magnetization curve for $U=0$ (circle), 1(triangle), 2(square) and 3(diamond).

FIG. 4. The energy gap for $U=0$ (circle), $U=1$ (triangle), 2(square) and 3(diamond) are plotted as a function of the magnetic field. The dotted lines indicate $E_{g}(h=0)-2 h$. The insert is the gap $2 \Delta_{1}$ obtained by Sugiyama, et al. for $\mathrm{YbB}_{12}$.

FIG. 5. The f-component magnetic susceptibility (filled circles) and that multiplied by $E_{g}$ (open circles) and by $z$ (square) scaled at $U=0$. 


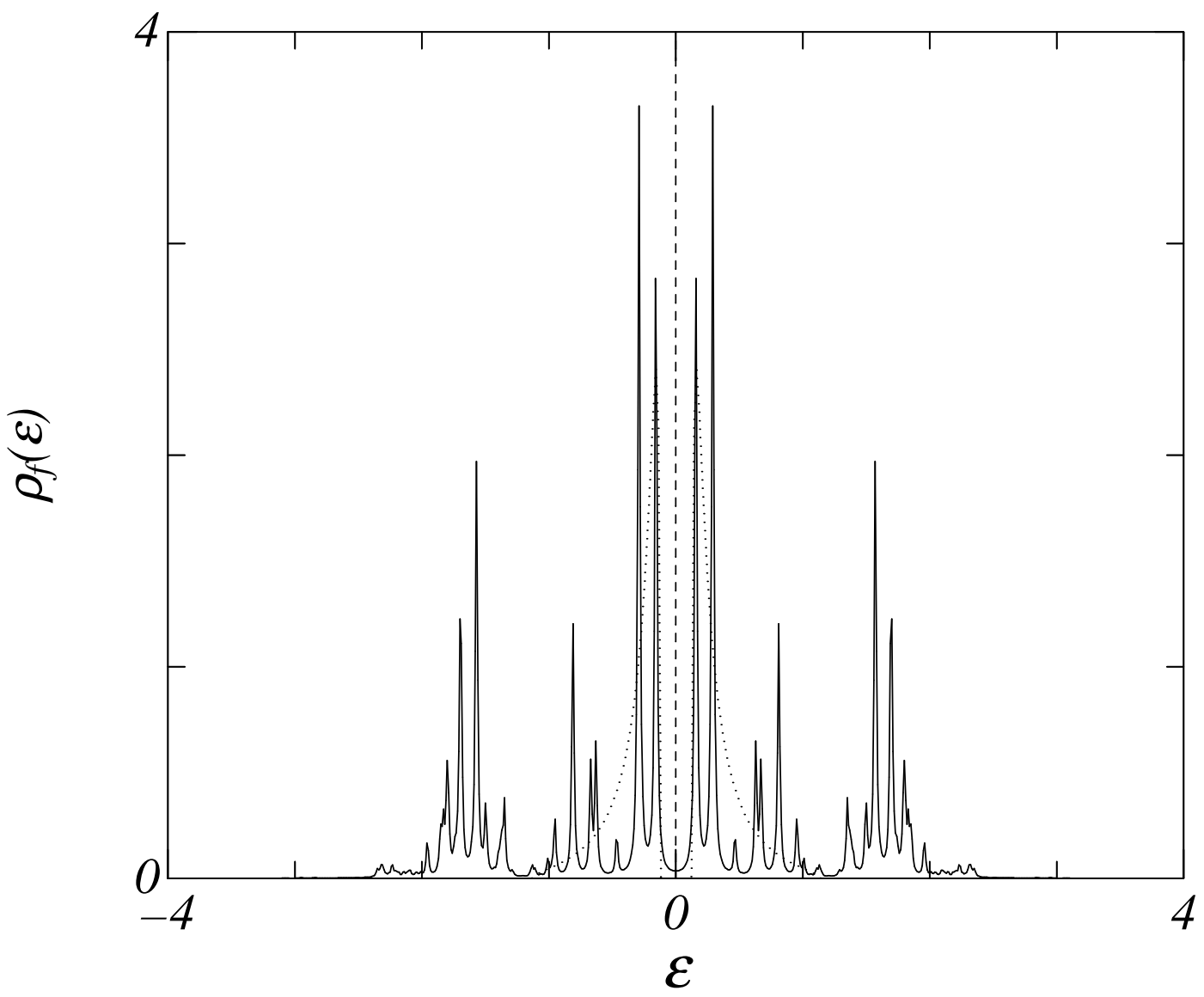




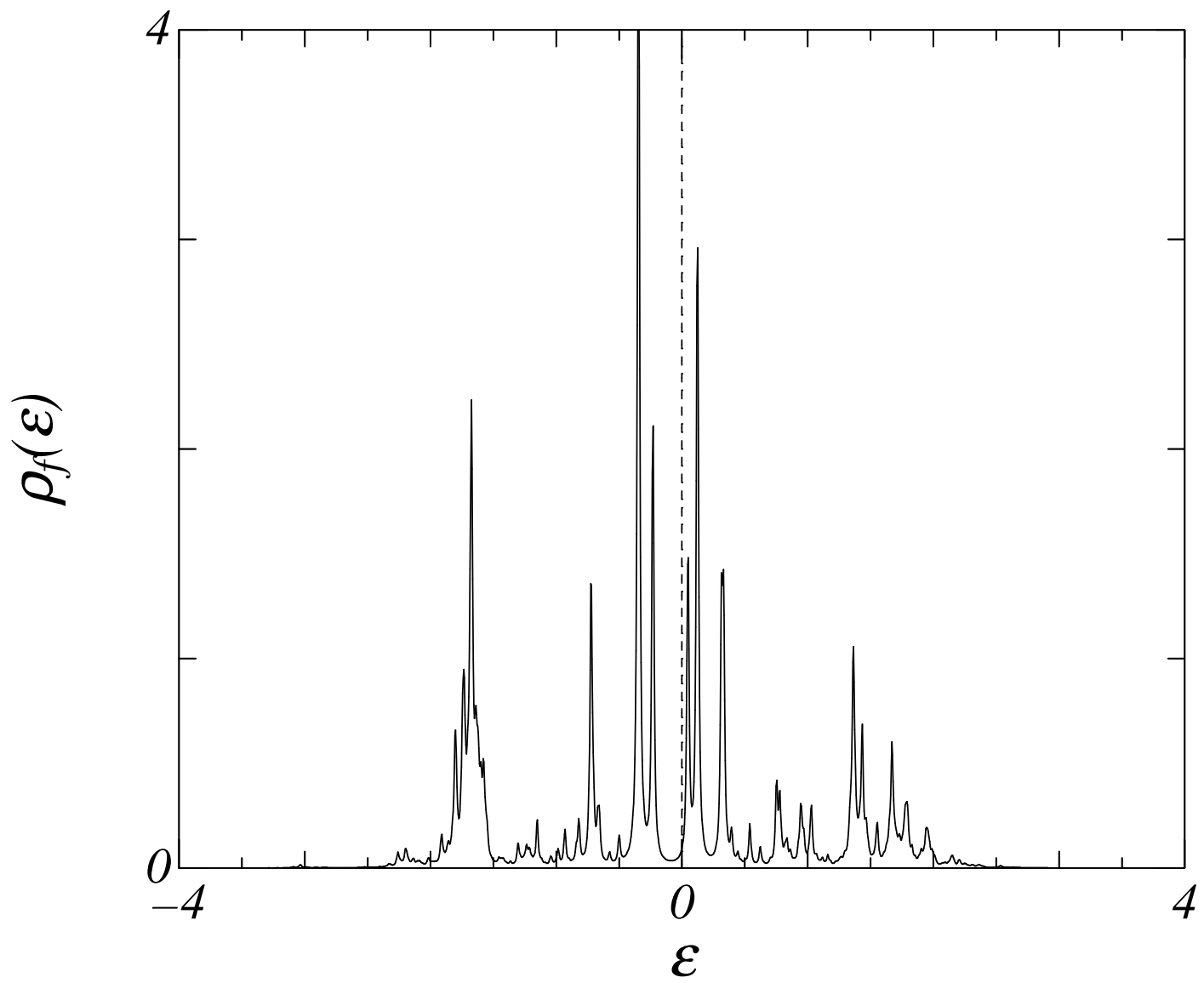




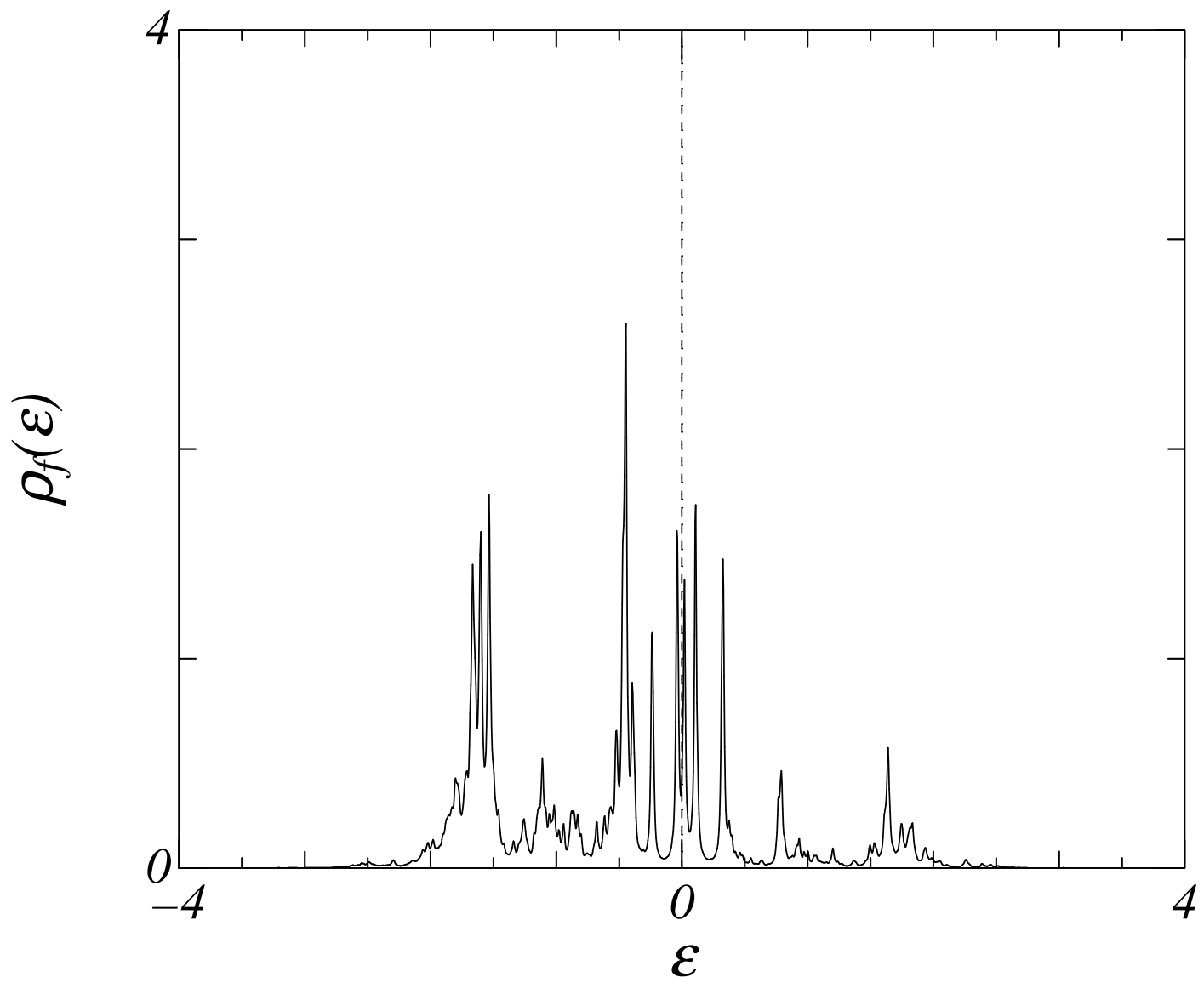




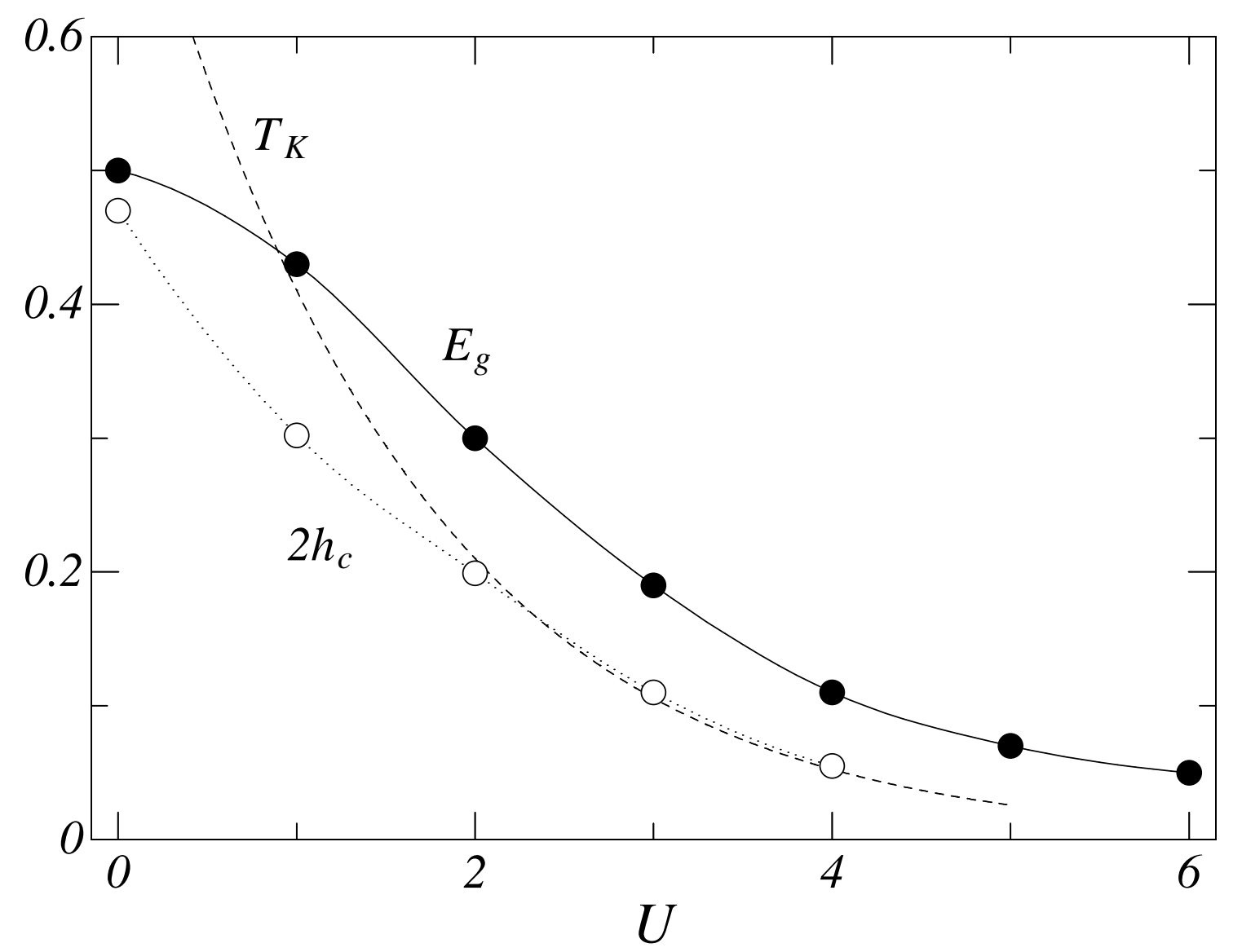




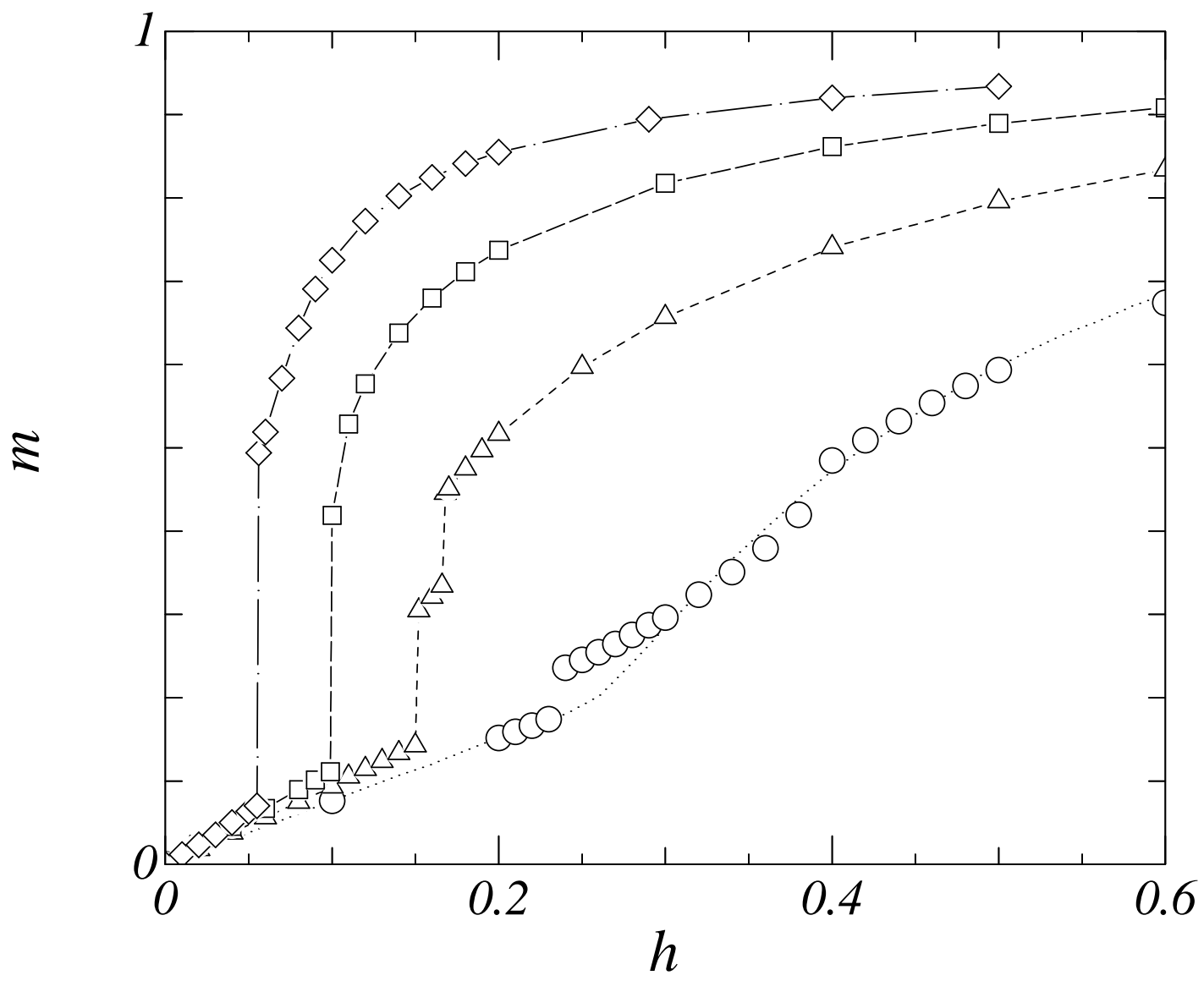




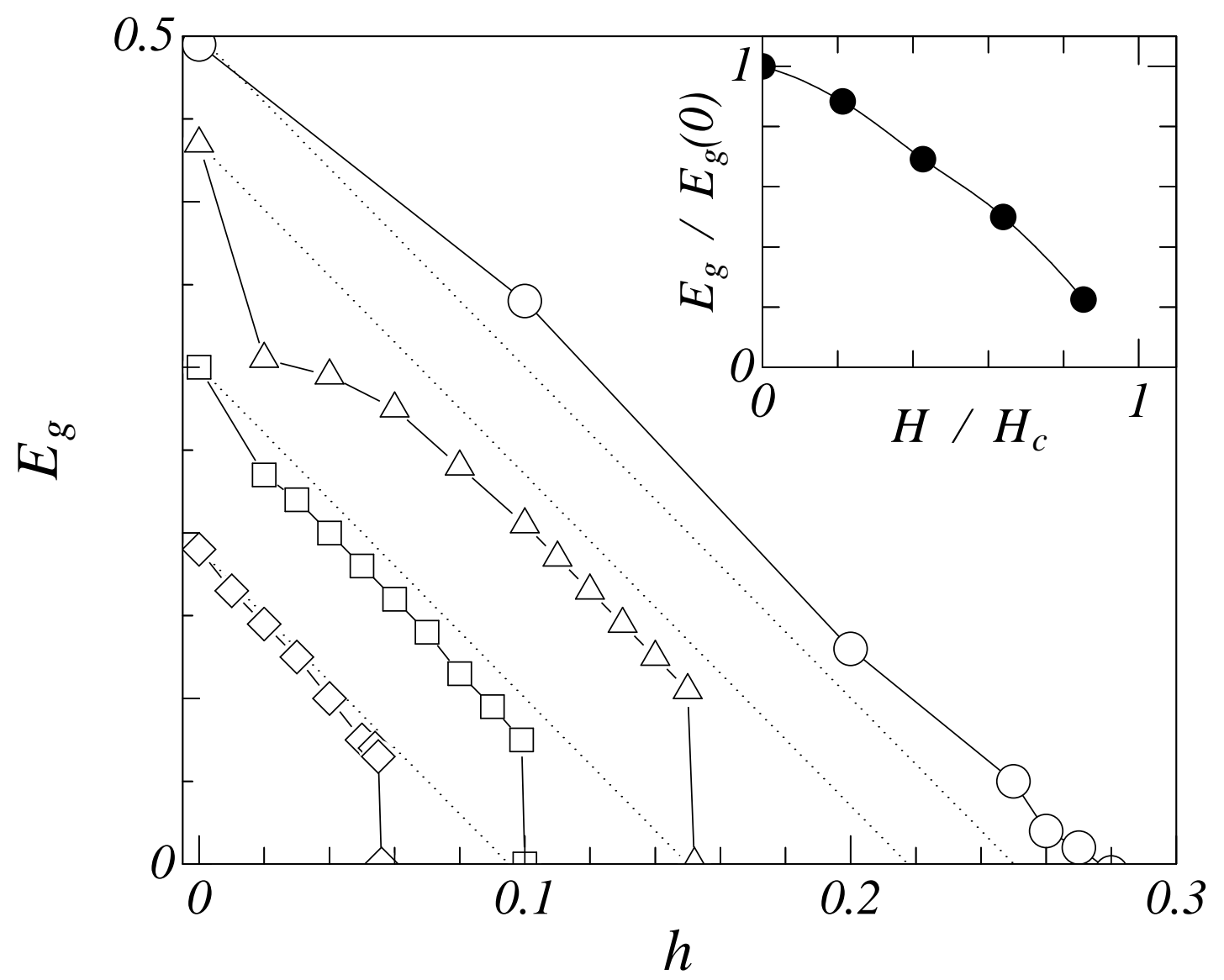




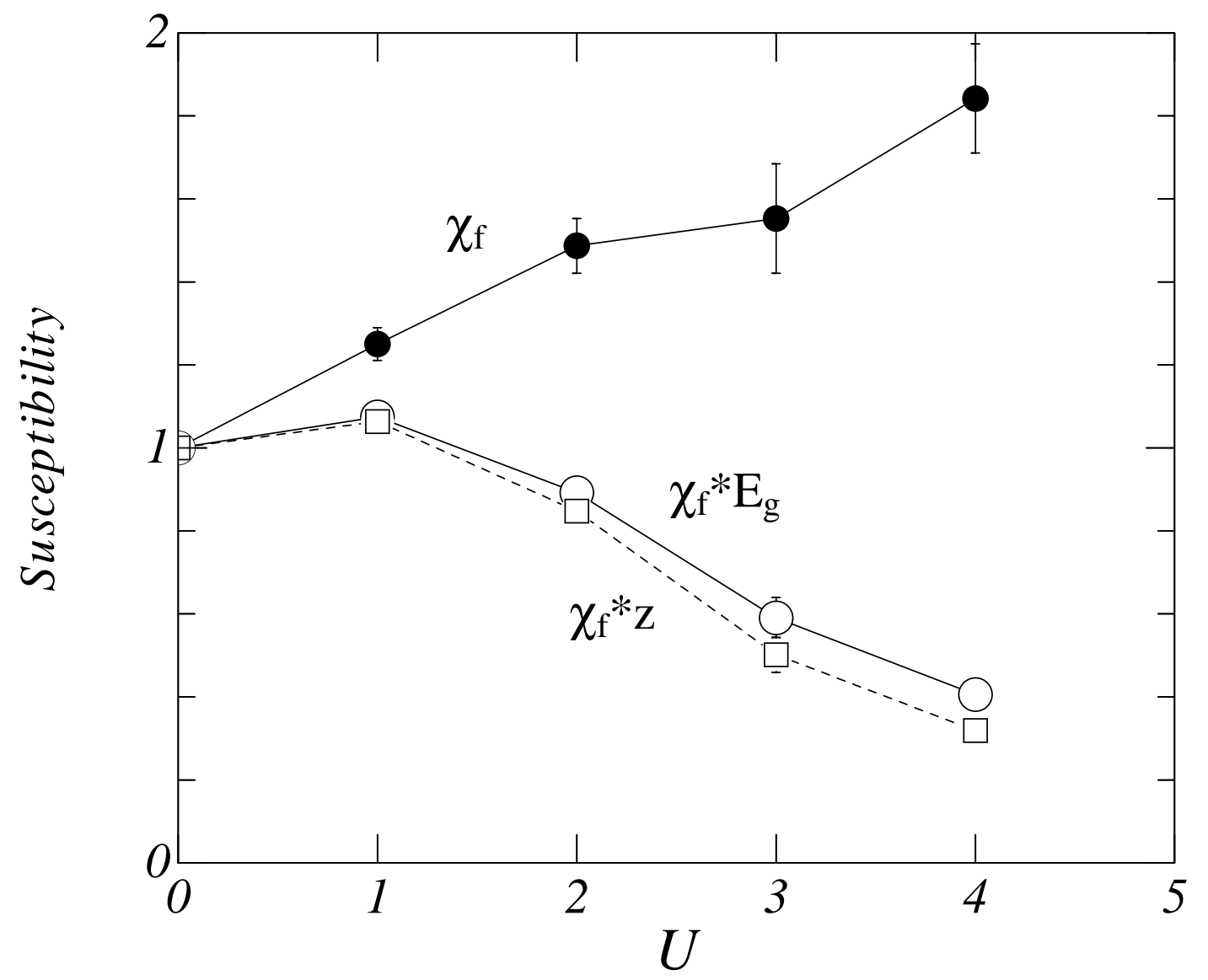

